$3-22-2003$

\title{
Small Angle Neutron Scattering from D2O-H2O Nanodroplets and Binary Nucleation Rates in A Supersonic Nozzle
}

Christopher H. Heath

Worcester Polytechnic Institute

Kiril A. Streletzky

Cleveland State University, K.STRELETZKY@csuohio.edu

Barbara E. Wyslouzil

Worcester Polytechnic Institute, barbaraw@wpi.edu

Judith Woelk

University of Cologne

Reinhard Strey

University of Cologne

Follow this and additional works at: https://engagedscholarship.csuohio.edu/sciphysics_facpub

Part of the Physics Commons

How does access to this work benefit you? Let us know!

Publisher's Statement

(C) 2003 American Institute of Physics.

\section{Repository Citation}

Heath, Christopher H.; Streletzky, Kiril A.; Wyslouzil, Barbara E.; Woelk, Judith; and Strey, Reinhard, "Small Angle Neutron Scattering from D2O-H2O Nanodroplets and Binary Nucleation Rates in A Supersonic Nozzle" (2003). Physics Faculty Publications. 257.

https://engagedscholarship.csuohio.edu/sciphysics_facpub/257

This Article is brought to you for free and open access by the Physics Department at EngagedScholarship@CSU. It has been accepted for inclusion in Physics Faculty Publications by an authorized administrator of EngagedScholarship@CSU. For more information, please contact library.es@csuohio.edu. 
Small angle neutron scattering from $\mathrm{D}_{2} \mathrm{O}-\mathrm{H}_{2} \mathrm{O}$ nanodroplets and binary nucleation rates in a supersonic nozzle

Christopher H. Heath, Kiril A. Streletzky, and Barbara E. WyslouzilJudith Wölk and Reinhard Strey

Citation: 118, 5465 (2003); doi: 10.1063/1.1554736

View online: http://dx.doi.org/10.1063/1.1554736

View Table of Contents: http://aip.scitation.org/toc/jcp/118/12

Published by the American Institute of Physics

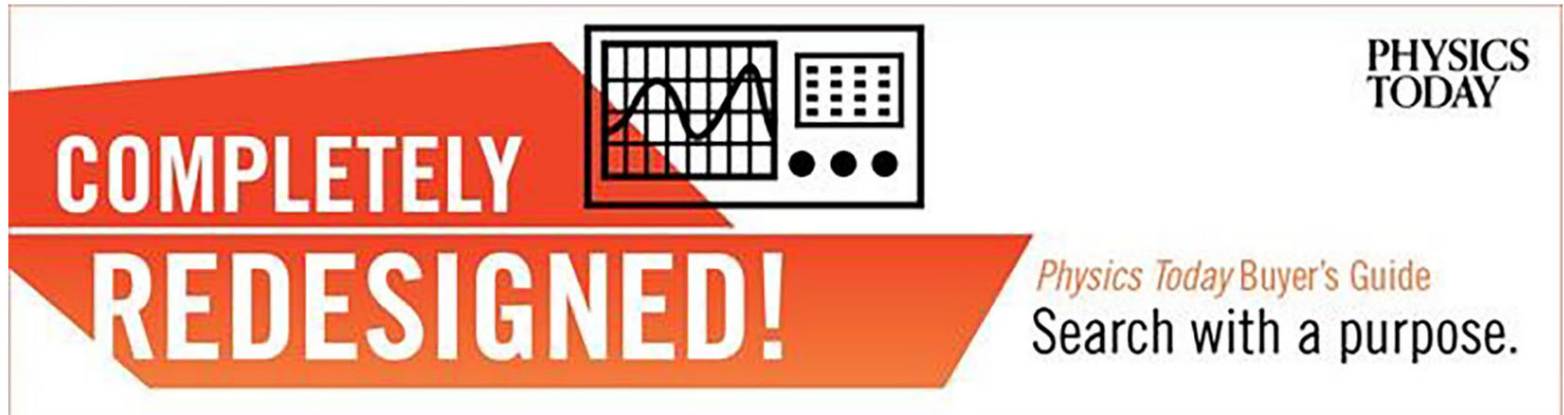




\title{
Small angle neutron scattering from $\mathrm{D}_{2} \mathrm{O}-\mathrm{H}_{2} \mathrm{O}$ nanodroplets and binary nucleation rates in a supersonic nozzle
}

\author{
Christopher H. Heath, Kiril A. Streletzky, and Barbara E. Wyslouzil ${ }^{\text {a) }}$ \\ Department of Chemical Engineering, Worcester Polytechnic Institute, Worcester, Massachusetts 01609-2280 \\ Judith Wölk and Reinhard Strey \\ Institut für Physikalische Chemie, Universität zu Köln, 50939 Köln, Germany
}

(Received 17 December 2002; accepted 3 January 2003)

\begin{abstract}
Small angle neutron scattering (SANS) experiments were used to characterize binary nanodroplets composed of $\mathrm{D}_{2} \mathrm{O}$ and $\mathrm{H}_{2} \mathrm{O}$. The droplets were formed by expanding dilute mixtures of condensible vapor in a $\mathrm{N}_{2}$ carrier gas through a supersonic nozzle, while maintaining the onset of condensation at a fixed position in the nozzle. It is remarkable, given the small coherent scattering length density of light water, that even the pure $\mathrm{H}_{2} \mathrm{O}$ aerosol gave a scattering signal above background. The scattering spectra were analyzed assuming a log-normal distribution of droplets. On average, the geometric radius of the nanodroplets $r_{g}$ was $r_{g}=13( \pm 1) \mathrm{nm}$, the polydispersity $\ln \sigma_{r}$ was $\ln \sigma_{r}$ $=0.19( \pm 0.07)$, and the number density $N$ was $N=(2 \pm 0.2) \cdot 10^{11} \mathrm{~cm}^{-3}$. The aerosol volume fractions derived from the SANS measurements are consistent with those derived from the pressure trace experiments, suggesting that the composition of the droplets was close to that of the initial condensible mixture. A quantitative analysis of the scattering spectra as a function of the isotopic composition gave further evidence that the binary droplets exhibit ideal mixing behavior. Because both the stagnation temperature $T_{0}$ and the location of onset were fixed, the temperature corresponding to the maximum nucleation rate was constant at $T_{J \max }=229( \pm 1) \mathrm{K}$. Thus, the experiments let us estimate the isothermal peak nucleation rates as a function of the isotopic composition. The nucleation rates were found to be essentially constant with $J_{\max }$ equal to (3.6 $\pm 0.5) \cdot 10^{16} \mathrm{~cm}^{-3} \mathrm{~s}^{-1}$ at a mean supersaturation of $44( \pm 3)$. (C) 2003 American Institute of Physics. [DOI: 10.1063/1.1554736]
\end{abstract}

\section{INTRODUCTION}

Multicomponent nanometer sized droplets form both in the environment and in large-scale industrial processes. Accurate predictions of the rate at which the phase transitions occur, and the structure of the final droplets are critical for developing reliable models of industrial processes, climate, and atmospheric chemistry. The nucleation rate directly affects the aerosol size distribution and, thus, the surface area available for heterogeneous reaction. Differences between the surface and interior compositions, on the other hand, affect heterogeneous chemistry, growth, and evaporation kinetics and even the nucleation rate of the droplets themselves. Unlike solid particles which can be captured and subjected to further analysis, liquid droplets must be examined in situ.

Small angle neutron scattering (SANS) has been successfully used to examine the structure of matter in the $1-100 \mathrm{~nm}$ range. It is routinely used to study the properties of complex fluids such as microemulsions ${ }^{1-3}$ and polymer solutions. ${ }^{4}$ Despite over half a century of application, it has only recently been used to study aerosols. ${ }^{5-7}$ Although aerosol-SANS experiments are difficult, they are already yielding unique results regarding droplet formation rates and nanodroplet microstructure.

To generate a nanodroplet aerosol with a volume fraction

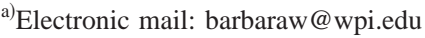

high enough to produce a detectable SANS scattering signal, we work with supersonic nozzles. Expansion rates in these devices are on the order of $p^{-1} d p / d t=10^{4} \mathrm{~s}^{-1}$, and the supersaturation reached by either isotope of water can be as high as several hundred. ${ }^{8}$ The aerosol typically has a number density of $10^{11} \mathrm{~cm}^{-3}$, a median radius $r_{g}$ between 4 and $15 \mathrm{~nm}$, and a polydispersity of 0.1 to $0.3 .^{5^{5}-7}$

In this paper we present the results of our first systematic SANS study of multicomponent droplets. We worked with $\mathrm{D}_{2} \mathrm{O}-\mathrm{H}_{2} \mathrm{O}$ mixtures because in this highly ideal system the assumption that the mole fraction of $\mathrm{D}_{2} \mathrm{O}$ in the nanodroplets is close to that of the liquid used to generate the condensible vapor is quite reasonable even if not all of the material has condensed. The surface tensions of $\mathrm{D}_{2} \mathrm{O}$ and $\mathrm{H}_{2} \mathrm{O}$ are also very close and, thus, the droplets themselves should not exhibit any microstructure. On the other hand, the scattering length density is a strong function of the composition of the droplets, and so the scattering spectra will change dramatically as the $\mathrm{D}_{2} \mathrm{O}$ content of the droplets changes. Although interpreting the spectra of multicomponent droplets is more complex than interpreting the spectra of droplets containing only one species, assuming that condensation proceeds via a $\mathrm{D}_{2} \mathrm{O}-\mathrm{H}_{2} \mathrm{O}$ pseudocomponent simplifies the analysis and is a reasonable first step. In fact, the rapid isotopic exchange that occurs between $\mathrm{H}_{2} \mathrm{O}$ and $\mathrm{D}_{2} \mathrm{O}$ means that $\mathrm{HDO}$ is often the dominant species. Finally, by analyzing the static pressure profiles, we can estimate the characteristic time, temperature, 
and supersaturation corresponding to the maximum nucleation rate during the expansion. Combining this information with an estimate for the aerosol number density from SANS lets us make the first estimates of binary nucleation rates in a supersonic nozzle. With these estimates we can test whether the constant onset isotherms for $\mathrm{D}_{2} \mathrm{O}-\mathrm{H}_{2} \mathrm{O}$ mixtures correspond to the constant nucleation rate isotherms produced in expansion cloud chambers and other nucleation devices.

The paper is organized as follows: In the Experiment section we briefly describe the supersonic nozzle, the setup for the SANS measurements, and how nucleation rates can be obtained from the data. In the Results section we present the main experimental results, namely the SANS curves and the aerosol number densities derived from them. In the Discussion section these data are used to estimate the homogeneous nucleation rates for both isotopes of water and four intermediate mixtures. The paper concludes by comparing these nucleation rates with the predictions of correlations based on nucleation pulse chamber experiments.

\section{EXPERIMENT}

\section{A. Materials and physical properties}

Small angle neutron scattering experiments are greatly facilitated if compounds containing deuterium instead of hydrogen are used. The $\mathrm{D}_{2} \mathrm{O}$ (Sigma Aldrich) had more than 99.9\% D substitution. The $\mathrm{H}_{2} \mathrm{O}$ was deionized and had a resistivity greater than $15 \mathrm{M} \Omega \cdot \mathrm{cm}$. The resistivity of $\mathrm{D}_{2} \mathrm{O}$ was of a similar magnitude. We used the thermophysical parameters of light and heavy water presented by Wölk and Strey ${ }^{9}$ to invert the pressure trace data (cf. subsection C, below) and to calculate the theoretical nucleation rates. For the sake of completeness, their correlations for surface tension $\sigma$, the vapor pressure $p^{\infty}(T)$, the density $\rho$, and compressibility $\kappa$ are summarized in the Appendix. The heat of vaporization values are derived from the equilibrium vapor pressures using the Clausius-Clapeyron equation.

\section{B. Supersonic nozzle}

The supersonic nozzle apparatus used to produce the aerosols has been described extensively elsewhere. ${ }^{8,10}$ To summarize briefly, we generated a gas stream that consists of $\mathrm{N}_{2}$ and up to $2.5 \mathrm{~mol} \%$ of the condensible vapor of interest. The stagnation temperature $T_{0}$ of the mixed stream was close to room temperature, and the initial supersaturation $S_{0}$ of the condensible vapor was as high as 0.45 . As the mixture expands in the nozzle, the temperature drops at a rate of about $10^{6} \mathrm{~K} / \mathrm{s}$ and the condensible vapor becomes highly supersaturated. Most of the droplets form in a rapid burst of nucleation that lasts from 10 to $50 \mu \mathrm{s}$. Modeling and very recent experimental work both show that the peak nucleation rates are about $10^{15}-10^{8} \mathrm{~cm}^{-3} \mathrm{~s}^{-1}$, depending on the particular nozzle design. The droplets formed in the nucleation burst grow rapidly, consume the condensible vapor, and quench further particle formation. The vapor to liquid phase transition releases heat to the flow, thereby increasing the pressure of the system above that expected for an isentropic expansion of the same gas mixture. Measuring the pressure as a function of position in the nozzle is a convenient way to detect and follow the entire condensation process.

\section{Pressure measurements}

Before conducting the SANS experiments, we performed extensive pressure trace experiments to characterize the condensation of $\mathrm{H}_{2} \mathrm{O}-\mathrm{D}_{2} \mathrm{O}$ mixtures in the nozzle as a function of the initial gas composition and stagnation temperature $T_{0}$. All expansions started from a stagnation pressure of $p_{0}=59.6 \pm 0.1 \mathrm{kPa}$. We first determined the shape of the nozzle by measuring the static pressure profile for the flow of a pure carrier gas. We then measured the static pressure profiles for different initial partial pressures of the condensible vapor, $p_{\nu, 0}=p_{\mathrm{D}_{2} \mathrm{O}}+p_{\mathrm{H}_{2} \mathrm{O}}$, at mole fractions of $\mathrm{D}_{2} \mathrm{O}$ in the condensible vapor $\tilde{y}=p_{\mathrm{D}_{2} \mathrm{O}} /\left(p_{\mathrm{D}_{2} \mathrm{O}}+p_{\mathrm{H}_{2} \mathrm{O}}\right)$ equal to 1.0, 0.8, 0.6, 0.4, 0.2, and 0.0. Here, $p_{\mathrm{D}_{2} \mathrm{O}}$ and $p_{\mathrm{H}_{2} \mathrm{O}}$ are the partial pressures of $\mathrm{D}_{2} \mathrm{O}$ and $\mathrm{H}_{2} \mathrm{O}$, respectively. To determine the other properties of the expansion, we integrated the diabatic flow equations using the measured area ratio of the nozzle, the condensing flow pressure trace, the stagnation conditions, and an equation of state. We defined the onset of condensation as that point in the flow where the difference in temperature between the condensing flow $T_{\mathrm{cf}}$ and the isentropic expansion of a gas with the same properties of the mixture $T_{\mathrm{mi}}$ is $0.5 \mathrm{~K}$. For a fixed value of $\tilde{y}$, the onset pressure $p_{\text {on }}$ is an exponential function of onset temperature $T_{\text {on }}$. From fits to the onset data we can determine the partial pressure of each species required to maintain onset at a constant temperature. When the experiments have the same value of $T_{0}$, constant onset temperature also corresponds to a fixed position for the onset of condensation in the nozzle. Thus, the expansions all experience the same gas dynamic history up to onset.

\section{Aerosol-SANS: Experimental setup and data analysis}

The experimental setup for the SANS measurements with the supersonic nozzle has been described in more detail in previous papers. ${ }^{5-7}$ Briefly, the SANS experiments were conducted using the NG-7 SANS instrument at the NIST Center for Neutron Research, Gaithersburg, MD. During a SANS experiment, the nozzle, plenum, and associated plumbing are placed in the sample chamber. The windows separating the sample chamber from the neutron guide and the detector tube are removed and the entire system is pumped down to about $12 \mathrm{~Pa}$. The neutron beam crosses the sample at right angles to the gas flow, and the $0.6 \mathrm{~cm}^{3}$ scattering volume is defined by the width of the nozzle $(1.27 \mathrm{~cm})$, and a $1.2 \mathrm{~cm}$ wide $\times 0.4 \mathrm{~cm}$ high cadmium aperture centered $5.6 \mathrm{~cm}$ downstream of the throat (cf. Fig. 2 in Ref. 5).

For these experiments the neutron scattering detector consisted of a $64 \times 64$ array of $1 \mathrm{~cm}^{2}{ }^{3} \mathrm{He}$ detectors. Scattering from the pure $\mathrm{D}_{2} \mathrm{O}(\tilde{y}=1.0)$ aerosol was measured at sample-to-detector distances (SDD) of 1.0 and $2.9 \mathrm{~m}$. For pure $\mathrm{H}_{2} \mathrm{O}(\tilde{y}=0.0)$ and the multicomponent aerosols $(\tilde{y}=0.8,0.6,0.4,0.2)$ only the $2.9 \mathrm{~m}$ SDD was used. The neutron wavelength $\lambda$ was $\lambda=0.8 \mathrm{~nm}$, corresponding to a 
mean neutron velocity of $500 \mathrm{~m} / \mathrm{s}$, and the wavelength spread was $\Delta \lambda / \lambda=22 \%$. The neutron scattering measurements were made in 30-min intervals with 30-min background measurements of the pure nitrogen gas before and after each aerosol measurement. The total integration time for each aerosol sample at each SDD was about $1 \mathrm{~h}$. By alternating sample and background measurements we could monitor and properly account for slight drifts in the background due to the build-up of contaminants on the windows. The two-dimensional data were treated to account for background scattering, sample transmission, pixel-to-pixel intensity variations, and bad regions of the detector. The absolute intensity scale was determined by measuring the attenuated neutron flux reaching the detector during a beam center determination.

The two-dimensional data were then averaged using the NIST data reduction software ${ }^{11}$ to produce the onedimensional scattering intensity $I(q)$, where $q$ is the momentum transfer wave vector. In supersonic nozzle experiments the droplets have a velocity in the flow direction that is comparable to the neutron velocity. Thus, the momentum transfer vector $q$ depends not only on the neutron wavelength and the normal scattering angle $\theta$, but also on the azimuthal angle on the detector and the ratio of the aerosol particle velocity to the neutron velocity. The necessary Doppler-shift correction was applied to the data ${ }^{11,12}$ during the averaging procedure. We use the velocity of the aerosol droplets derived from the pressure trace information and assume that the droplets move at the same speed as the gas. This is consistent with the velocity derived from Doppler anisotropy of the 2D spectra. ${ }^{6}$

The data were fit to extract the size parameters using the NIST analysis routines ${ }^{11}$ assuming a log-normal or Gaussian size distribution of droplets and taking the instrument resolution into account (i.e., smeared fits). The formal errors quoted for each parameter correspond to 1 standard deviation from the mean with all other parameters optimized.

\section{E. Nucleation rate estimates}

As discussed by Streletzky et al. ${ }^{7}$ and Khan et al. ${ }^{13,14}$ the nucleation rates in supersonic nozzles can be estimated directly by analyzing both the pressure trace data and the SANS experiments. The analysis is analogous to that used to determine nucleation rates in laminar diffusion flow tubes. ${ }^{15-17}$ We start by assuming that the ratio of the maximum nucleation rate $J^{\max }$ to the particle production rate $\int J d V$ is the same for the experiments as for a reasonable nucleation rate theory, i.e., that

$$
\frac{J_{\exp }^{\max }}{\int J_{\exp } d V}=\frac{J_{\text {theory }}^{\max }}{\int J_{\text {theory }} d V}=\frac{1}{V_{J_{\max }}},
$$

where $V_{J_{\max }}$ is the characteristic volume corresponding to the maximum nucleation rate. The characteristic time corresponding to the maximum rate is then given by

$$
\Delta t_{J_{\max }}=\frac{V_{J_{\max }}}{\dot{V}_{\mathrm{NZ}}},
$$

where $\dot{V}_{\mathrm{NZ}}$ is the volumetric flow rate through the nucleation zone. All of the information required to calculate $\Delta t_{J_{\max }}$ can be derived from the pressure trace measurements. The peak nucleation rate is then given by

$$
J_{\max }\left(S_{J_{\max }}, T_{J_{\max }}\right)=\frac{N}{\Delta t_{J_{\max }}} f_{\exp },
$$

where $N$ is the number density of the aerosol measured using SANS. The factor $f_{\text {exp }}$ corrects the observed number density for the continued expansion between the nucleation zone (NZ) and the viewing volume (VV), and is given by the density ratio $f_{\exp }=\rho_{\mathrm{NZ}} / \rho_{\mathrm{VV}}$. The values of $S_{J_{\max }}$ and $T_{J_{\max }}$ are those that maximize the theoretical nucleation rate, and these are also quite insensitive to the theory used.

\section{RESULTS}

\section{A. Pressure trace measurements}

The detailed results of the extensive pressure trace experiments made for the $\mathrm{D}_{2} \mathrm{O}-\mathrm{H}_{2} \mathrm{O}$ mixtures in this nozzle are presented in a previous paper. ${ }^{8}$ Here, we focus solely on the results relevant to the SANS experiments where all expansions started at $T_{0}=26.0 \pm 0.1^{\circ} \mathrm{C}$. We maintained onset about $1.2 \mathrm{~cm}$ downstream of the throat and, thus, the temperature at onset was close to $230 \mathrm{~K}$. This temperature is easily reached by other nucleation experiments, in particular expansion cloud chambers and shock tubes. Figure 1(a) illustrates the onset pressure as a function of the onset temperature measured after the SANS experiments were conducted. In all of these experiments, approximately $75 \%$ of the initial vapor condensed by the time the flow reached the viewing volume.

Although one nozzle sidewall was removed in order to replace a contaminated Si window after the SANS experiments were completed, we believe that the nozzle flow area was virtually the same for the SANS and the pressure trace experiments. Figure 1(b) summarizes the partial pressures of $\mathrm{D}_{2} \mathrm{O}$ and $\mathrm{H}_{2} \mathrm{O}$ at onset for the conditions used at NIST and compares them to the conditions that would have maintained onset at $T=230 \mathrm{~K}$. The agreement is quite good. Finally, Table I summarizes the important experimental parameters including the initial conditions, as well as those at onset and in the viewing volume. The results in Table I were derived from the pressure trace experiments by correlating each parameter as a function of the mass flow rate of the condensible vapor entering the system, and then interpolating or extrapolating to the flow rates used during the SANS experiments.

\section{B. Small angle neutron scattering}

The measured SANS scattering spectra are illustrated in Fig. 2 as a function of the momentum transfer vector $q$.

The raw scattering spectra, Fig. 2(a), graphically illustrate how close the scattering spectrum of the pure $\mathrm{H}_{2} \mathrm{O}$ aerosol lies to the scattering spectrum of the nozzle flowing pure $\mathrm{N}_{2}$. Figure 2(b) shows the background subtracted spectra and includes the best fits to the spectra assuming the aerosol has a log-normal size distribution. In both figures it is clear that the scattered neutron intensity is a strong function of the 

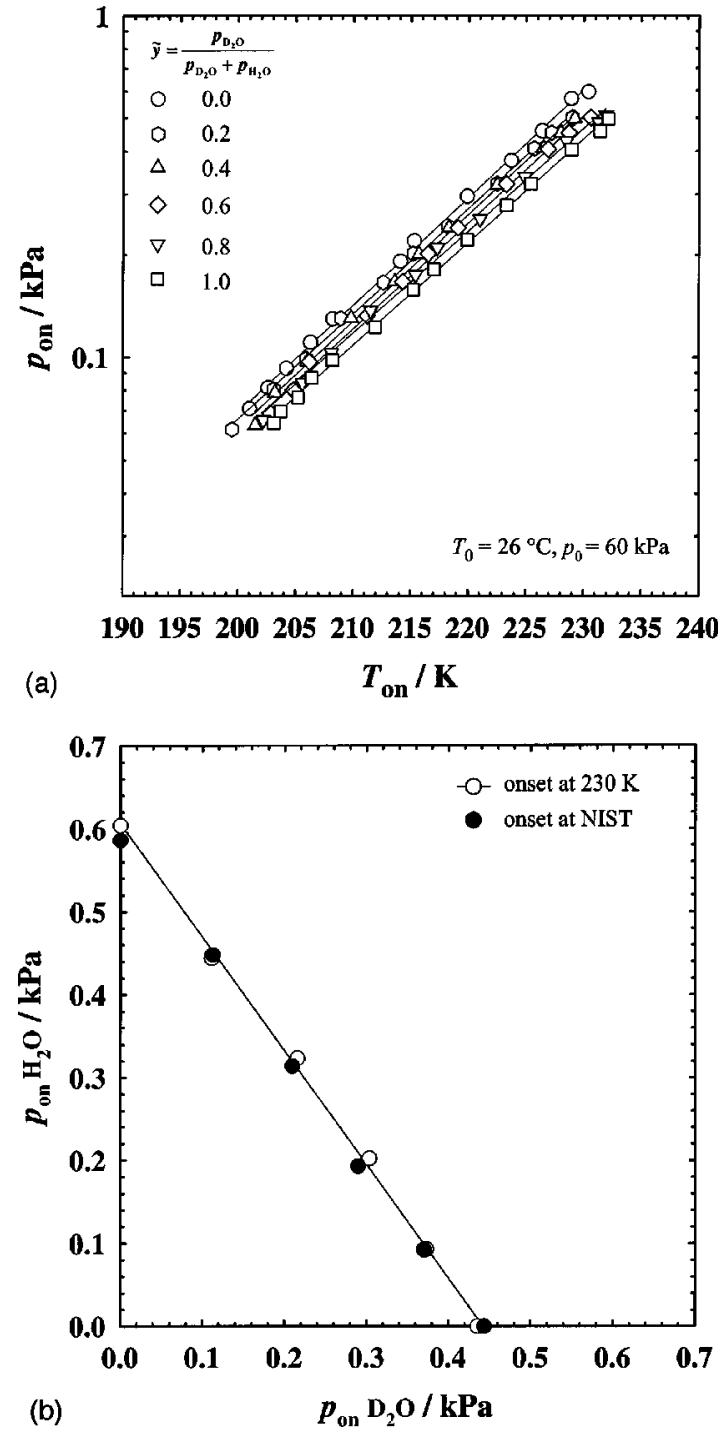

FIG. 1. (a) The Wilson plot for the experiments starting at $T_{0}=26.0$ $\pm 0.1^{\circ} \mathrm{C}$ and $p_{0}=59.6 \pm 0.1 \mathrm{kPa}$. (b) The partial pressures of $\mathrm{D}_{2} \mathrm{O}$ and $\mathrm{H}_{2} \mathrm{O}$ corresponding to the experiments at NIST are very close to those required to maintain onset at $230 \mathrm{~K}$

$\mathrm{D}_{2} \mathrm{O}$ mole fraction. This is because the absolute intensity of a scattering spectrum depends on the square of the difference in scattering length density between the scattering body and the solvent, $(\Delta \rho)^{2}$. The quantity $(\Delta \rho)^{2}$ is also called the

TABLE I. The parameters at onset and in the viewing volume are those that correspond to the SANS experiments. All expansions started from $p_{0}$ $=59.6 \pm 0.1 \mathrm{kPa}$ and $T_{0}=26.0 \pm 0.1^{\circ} \mathrm{C}$. Here, $\tilde{y}=p_{\mathrm{D}_{2} \mathrm{O}} /\left(p_{\mathrm{D}_{2} \mathrm{O}}+p_{\mathrm{H}_{2} \mathrm{O}}\right), \dot{m}$ is the mass flow rate of the condensible vapor, $p_{\text {on }}$ and $T_{\text {on }}$ are the partial pressure of the condensible and the temperature at onset, $T_{\mathrm{vv}}$ and $\nu_{\mathrm{vv}}$ are the average temperature and velocity in the viewing volume, respectively. Note, at onset $p_{\mathrm{D}_{2} \mathrm{O}}=\tilde{y} \cdot p_{\text {on }}$ and $p_{\mathrm{H}_{2} \mathrm{O}}=(1-\tilde{y}) \cdot p_{\text {on }}$.

\begin{tabular}{cccccc}
\hline \hline$\tilde{y}$ & $\dot{m} / \mathrm{g} \mathrm{min}^{-1}$ & $p_{\text {on }} / \mathrm{kPa}$ & $T_{\text {on }} / \mathrm{K}$ & $T_{\mathrm{vv}} / \mathrm{K}$ & $\nu_{\mathrm{vv}} / \mathrm{ms}^{-1}$ \\
\hline 1.0 & 6.47 & 0.444 & 230.3 & 231 & 435 \\
0.8 & 6.67 & 0.463 & 229.9 & 232 & 435 \\
0.6 & 6.86 & 0.483 & 229.3 & 233 & 435 \\
0.4 & 7.25 & 0.524 & 229.6 & 235 & 434 \\
0.2 & 7.51 & 0.561 & 230.1 & 236 & 433 \\
0.0 & 7.77 & 0.586 & 229.6 & 237 & 433 \\
\hline \hline
\end{tabular}
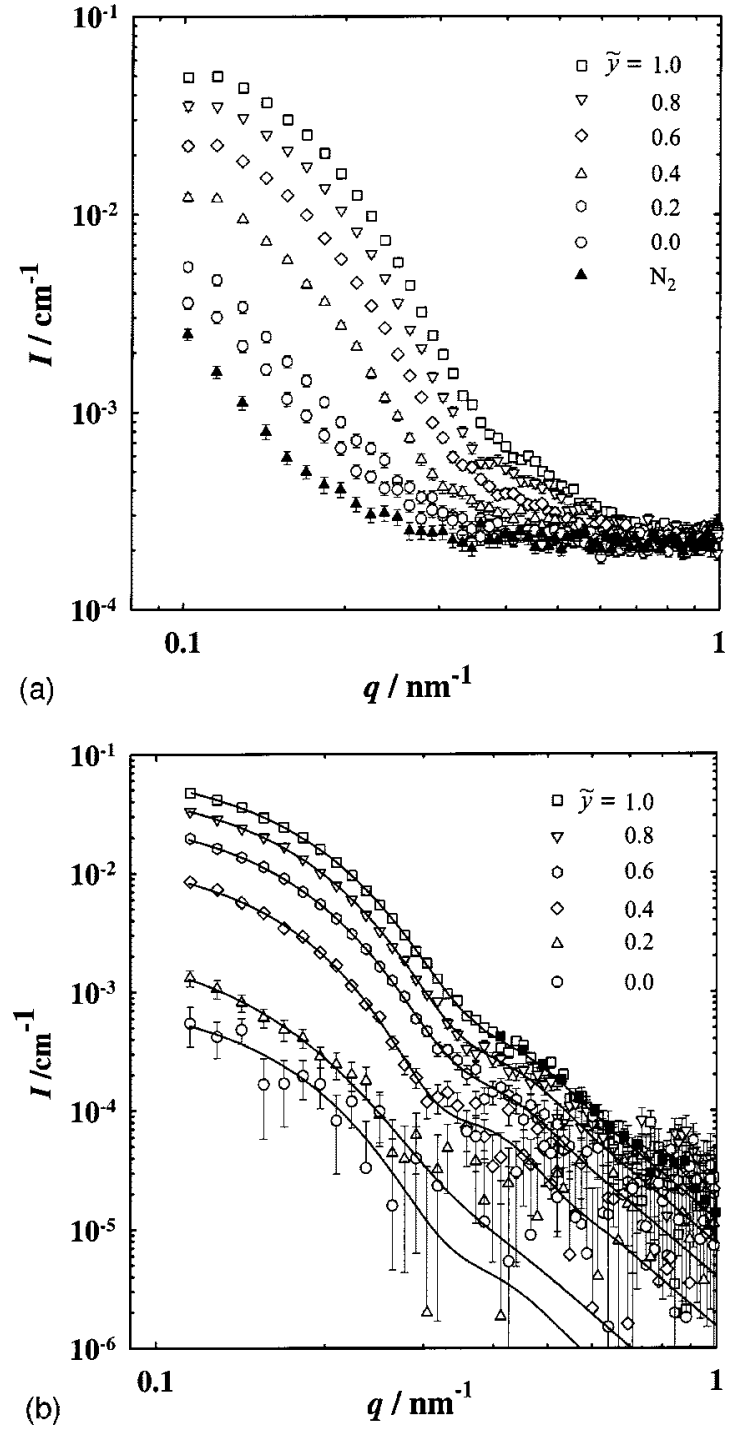

FIG. 2. The SANS scattering spectra for the $\mathrm{D}_{2} \mathrm{O}-\mathrm{H}_{2} \mathrm{O}$ aerosols. (a) The raw scattering spectra for the $\mathrm{D}_{2} \mathrm{O}-\mathrm{H}_{2} \mathrm{O}$ aerosols and from the flowing $\mathrm{N}_{2}$ carrier gas alone. (b) The background subtracted spectra. The mole fraction of $\mathrm{D}_{2} \mathrm{O}$ in the condensible vapor is given in the legend. Although the signal is weak, we were able to measure scattering from pure $\mathrm{H}_{2} \mathrm{O}$ aerosol.

contrast factor. In our case the solvent is the $\mathrm{N}_{2}$ carrier gas whose scattering length density is effectively zero, because the gas density is much lower than that of the liquid droplets. Thus,

$$
\Delta \rho=\rho_{\mathrm{D}_{2} \mathrm{O}} x+\rho_{\mathrm{H}_{2} \mathrm{O}}(1-x),
$$

where $x$ is the mole fraction of $\mathrm{D}_{2} \mathrm{O}$ in the droplets, and $\rho_{i}$ is the scattering length density of component $i$. The scattering length density of $\mathrm{D}_{2} \mathrm{O}$ is $\rho_{\mathrm{D}_{2} \mathrm{O}}=6.39 \cdot 10^{10} \mathrm{~cm}^{-2}$, while that of $\mathrm{H}_{2} \mathrm{O}$ is $\rho_{\mathrm{H}_{2} \mathrm{O}}=-0.56 \cdot 10^{10} \mathrm{~cm}^{-2}$, so even low levels of $\mathrm{H}_{2} \mathrm{O}$ can rapidly reduce the intensity of scattering signal from the multicomponent aerosol over that of the pure $\mathrm{D}_{2} \mathrm{O}$ aerosol. These values of scattering length densities assume that the density of $\mathrm{D}_{2} \mathrm{O}$ and $\mathrm{H}_{2} \mathrm{O}$ in the nanodroplets at the conditions in the viewing volume are 1.11 and $1.00 \mathrm{~g} \mathrm{~cm}^{-3}$, respectively, i.e., that the decrease in density of the liquids as the temperature decreases is compensated by the increase in density due to the high internal pressure of the droplets. The 
TABLE II. The properties of the aerosol size distributions were derived by fitting each spectrum to a log-normal size distribution. The value of $N$ in column 6 corresponds to fixing the value $\Delta \rho$ and optimizing $\phi_{\text {SANS }}$. The value of $N$ in column 9 corresponds to fixing the value of $\phi$ to $\phi_{\mathrm{PT}}$ and optimizing $\Delta \rho$. The values of $r_{\mathrm{g}}$ and $\ln \sigma_{\mathrm{r}}$ are independent of the assumptions regarding $\Delta \rho$ and $\phi$.

\begin{tabular}{|c|c|c|c|c|c|c|c|c|}
\hline$\tilde{y}$ & $r_{\mathrm{g}} / \mathrm{nm}$ & $\ln \sigma_{\mathrm{r}}$ & $\Delta \rho / \mathrm{cm}^{-2}$ & $\phi_{\text {SANS }}$ & $\mathrm{N} / \mathrm{cm}^{-3}$ & $\Delta \rho / \mathrm{cm}^{-2}$ & $\phi_{\mathrm{PT}}$ & $N / \mathrm{cm}^{-3}$ \\
\hline 1.0 & $12.2 \pm 0.1$ & $0.17 \pm 0.01$ & $6.39 \cdot 10^{10}$ & $(1.90 \pm 0.01) \cdot 10^{-6}$ & $2.16 \cdot 10^{11}$ & $(5.77 \pm 0.02) \cdot 10^{10}$ & $2.33 \cdot 10^{-6}$ & $2.64 \cdot 10^{11}$ \\
\hline 0.8 & $12.8 \pm 0.1$ & $0.16 \pm 0.01$ & $4.97 \cdot 10^{10}$ & $(2.06 \pm 0.02) \cdot 10^{-6}$ & $2.08 \cdot 10^{11}$ & $(4.61 \pm 0.02) \cdot 10^{10}$ & $2.39 \cdot 10^{-6}$ & $2.42 \cdot 10^{11}$ \\
\hline 0.6 & $13.2 \pm 0.2$ & $0.17 \pm 0.01$ & $3.59 \cdot 10^{10}$ & $(2.15 \pm 0.02) \cdot 10^{-6}$ & $1.98 \cdot 10^{11}$ & $(3.36 \pm 0.02) \cdot 10^{10}$ & $2.45 \cdot 10^{-6}$ & $2.25 \cdot 10^{11}$ \\
\hline 0.4 & $14.3 \pm 0.3$ & $0.13 \pm 0.02$ & $2.20 \cdot 10^{10}$ & $(2.28 \pm 0.04) \cdot 10^{-6}$ & $1.74 \cdot 10^{11}$ & $(2.09 \pm 0.02) \cdot 10^{10}$ & $2.57 \cdot 10^{-6}$ & $1.93 \cdot 10^{11}$ \\
\hline 0.2 & $12 \pm 2$ & $0.25 \pm 0.1$ & $0.82 \cdot 10^{10}$ & $(2.6 \pm 0.2) \cdot 10^{-6}$ & $2.37 \cdot 10^{11}$ & $(0.81 \pm 0.03) \cdot 10^{10}$ & $2.66 \cdot 10^{-6}$ & $2.40 \cdot 10^{11}$ \\
\hline 0.0 & $13 \pm 3$ & $0.17 \pm 0.2$ & $-0.56 \cdot 10^{10}$ & $(2.3 \pm 0.4) \cdot 10^{-6}$ & $2.03 \cdot 10^{11}$ & $(-0.51 \pm 0.04) \cdot 10^{10}$ & $2.74 \cdot 10^{-6}$ & $2.37 \cdot 10^{11}$ \\
\hline
\end{tabular}

error introduced by this assumption is of the order of $1 \%$ in the scattering length density and is therefore insignificant. Comparing the two extremes, a pure $\mathrm{D}_{2} \mathrm{O}$ aerosol has a scattered intensity $\sim 130$ times that of an identical pure $\mathrm{H}_{2} \mathrm{O}$ aerosol.

All of the scattering spectra in Fig. 2(b) have essentially the same shape. As $q$ approaches zero, the spectra begin to level off. For $0.3<q / \mathrm{nm}^{-1}<0.4$, all spectra have an inflection point in the region where the scattering amplitude for a single sphere crosses zero. ${ }^{18}$ The position of this minimum is related to the characteristic diameter of the droplets by

$$
\langle r\rangle=\frac{4.493}{q_{1}} .
$$

Thus, the systematic shift of the inflection points to smaller $q$ as the $\mathrm{H}_{2} \mathrm{O}$ level increases, indicates that the average droplet size is getting bigger. This behavior is consistent with the onset measurements, where the partial pressure to maintain onset at a fixed position increases with the $\mathrm{H}_{2} \mathrm{O}$ mole fraction. As $q$ increases beyond the inflection point, the scattering signal falls off as $q^{-4}$, consistent with scattering from homogeneous spherical droplets.

\section{Data analysis}

To determine the parameters of the size distribution, we assumed that the aerosol consists of a log-normal distribution of spherical droplets. In this case, the synthetic scattering spectrum for an aerosol with volume fraction $\phi$ is given by

$$
\begin{aligned}
I_{s}(q)= & \frac{3 \phi}{4 \pi\left\langle r^{3}\right\rangle} \cdot \frac{1}{\ln \left(\sigma_{r}\right) \sqrt{2 \pi}} \\
& \times \int_{0}^{\infty} \frac{1}{r} \exp \left[-\frac{\left(\ln r-\ln r_{g}\right)^{2}}{2 \ln ^{2} \sigma_{r}}\right] P(q, r) d r .
\end{aligned}
$$

Here, $r$ is the droplet radius, $r_{g}$ is the median or geometric droplet radius, $\ln \sigma_{r}$ is the polydispersity, and $\left\langle r^{3}\right\rangle$ is the third moment of $r$. The function $P(q, r)$ is the particle form factor for spherical droplets, and is given by

$$
P(q, r)=(\Delta \rho)^{2}\left[\frac{4 \pi(\sin (q r)-q r \cos (q r))}{q^{3}}\right]^{2} .
$$

In Eq. (6) $r_{g}$ and $\ln \sigma_{r}$ determine the shape of the scattering curve, while the product $\phi(\Delta \rho)^{2}$ determines the scaling. Because $\phi$ and $\Delta \rho$ are perfectly correlated, one of these parameters must be fixed when fitting the spectra while the other floats. When determining the best fit values of $\phi$ (or $\Delta \rho), r_{g}$, and $\ln \sigma_{r}$, the NIST curve-fitting program accounts for both the uncertainties associated with the experimental data and the instrument resolution.

We first fit the spectra assuming that the scattering length density and the absolute calibration of the SANS instrument are both correct (i.e., $\Delta \rho$ fixed) and found the best-fit values of $\phi, r_{g}$, and $\ln \sigma_{r}$ [Fig. 2(b)]. For a log-normal distribution of droplets, the value of $N$ is related to the volume fraction of condensate as

$$
N=\frac{3 \phi_{\text {SANS }}}{4 \pi\left\langle r^{3}\right\rangle}=\frac{3 \phi_{\text {SANS }}}{4 \pi r_{g}^{3}} \exp \left(-4.5 \ln ^{2} \sigma_{r}\right),
$$

where the subscript SANS has been added to emphasize that this is the value of $\phi$ derived purely from the SANS measurements. We also have an independent determination of volume fraction from the pressure trace measurements $\phi_{\mathrm{PT}}$. As summarized in Table II, the ratio $\phi_{\mathrm{SANS}} / \phi_{\mathrm{PT}}$ varies from 0.82 to 0.97 .

If, on the other hand, we insist that the volume fractions derived from the pressure traces are correct, then $\Delta \rho$ is $3 \%-$ $10 \%$ lower than the values used above to maintain a good fit. Physically, the uncertainty in the value of $\Delta \rho$ stems from uncertainty in scattering length density of the droplets and/or the instrument calibration. We note that the second method is not necessarily more accurate than the first because the values of $\phi_{\mathrm{PT}}$ also have uncertainty due both to the experimental measurements and the input parameters to the data inversion code, in particular the heat of vaporization of the water below $240 \mathrm{~K}$. The number densities, derived using Eq. (8), scale directly with $\phi$.

Table II summarizes the parameters of the size distributions derived using either approach. The formal errors in the fit parameters correspond to one standard deviation in the value of each parameter with the other parameters held at their optimal values. Although we do not report the results here, we also fit the data assuming Gaussian droplet distributions. Because the aerosols are reasonably monodisperse, we found little difference between the values derived for the 


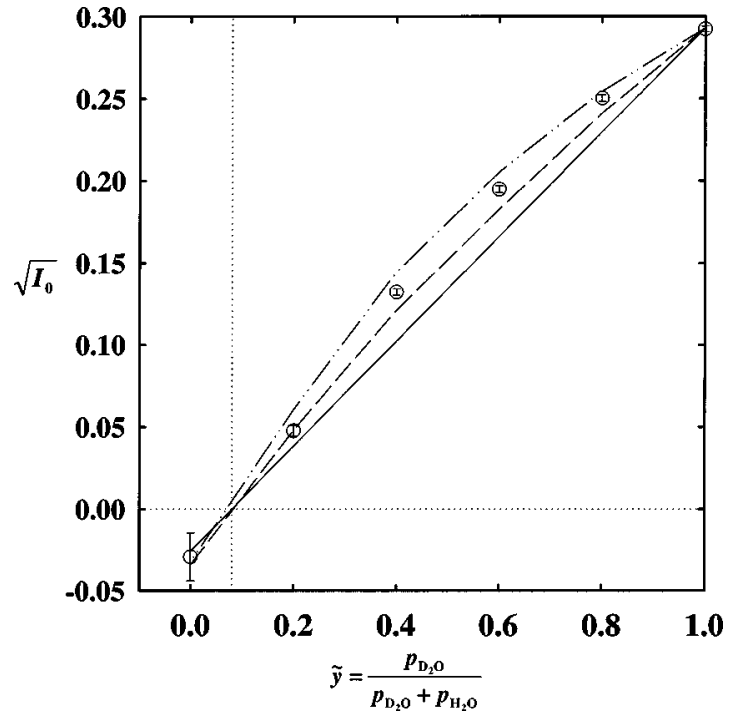

FIG. 3. The values of $\sqrt{I_{0}}$ obtained from Guinier fits to the data decrease rapidly as the $\mathrm{D}_{2} \mathrm{O}$ mole fraction in the condensible vapor is decreased. The solid line is the value of $\sqrt{I_{0}}$ for the pure $\mathrm{D}_{2} \mathrm{O}$ aerosol scaled by the mixture scattering length. The long dashed line corrects $\sqrt{I_{0}}$ for the change in particle size by scaling the solid line by $\dot{V}_{\text {mix }} / \dot{V}_{\text {D2O }}$. The dot-dash line corrects $\sqrt{I_{0}}$ for changes in particle size and polydispersity by scaling by $\left\langle r_{\text {mix }}^{6}\right\rangle /\left\langle r_{\mathrm{D} 2 \mathrm{O}}^{6}\right\rangle$. The dotted lines indicate zero scattering intensity and the mole fraction of the null contrast mixture, respectively. The error bars are statistical error for $I_{0}$ of the Guinier analysis, except for pure $\mathrm{H}_{2} \mathrm{O}$, where we averaged the error of the first three data points [cf. Fig. 2(b)].

median radius or the polydispersity (Gaussian versus lognormal), and in both cases these two parameters are highly correlated. Generally, the values of $N$ derived assuming a Gaussian size distribution were about 3\% higher than the values for the corresponding log-normal distribution.

\section{DISCUSSION}

\section{A. Total contrast and size distribution}

All of our analysis assumes that the $\mathrm{D}_{2} \mathrm{O}$ mole fraction in the droplets $x$ is equal to that of the initial condensible vapor mixture in the gas stream, $\widetilde{y}$. We can test this assumption by performing a simple Guinier analysis ${ }^{19}$ because the scattering intensity at $q=0, I_{0}$, depends quadratically on the contrast factor

$$
I_{0}=N\left\langle r^{6}\right\rangle(\Delta \rho)^{2},
$$

and the latter is a linear function of $x$.

In Fig. 3 we therefore plot the $\sqrt{I_{0}}$ as a function of the mole fraction of $\mathrm{D}_{2} \mathrm{O}$. For identical droplet size distributions, $\sqrt{I_{0}}$ should vary linearly with the mole fraction of $\mathrm{D}_{2} \mathrm{O}$, because $\Delta \rho$ is a linear function of $x$. The solid line in Fig. 3 illustrates the expected linear behavior of $\sqrt{I_{0}}$ where all the values are scaled to $I_{0}$ for the pure $\mathrm{D}_{2} \mathrm{O}$ aerosol. Although the experimental values of $\sqrt{I_{0}}$ change systematically with $x$, they almost all lie distinctly above the solid straight line.

It is already clear from the spectra in Fig. 2(b) and the phase behavior in Fig. 1 that the assumption of identical droplet distributions cannot be correct. The former shows that the inflection point, indicative of the average particle size, is changing as a function of composition, while the latter demonstrates that the partial pressure to maintain onset at a fixed position increases as mixtures become richer in $\mathrm{H}_{2} \mathrm{O}$. Since the molar densities of liquid $\mathrm{H}_{2} \mathrm{O}$ and $\mathrm{D}_{2} \mathrm{O}$ are essentially identical, the increase in partial pressure is achieved experimentally by increasing the volume of liquid that is pumped into the vaporizer. As noted earlier, we condense about $75 \%$ of the incoming material in each case. Thus, if the number density of each aerosol is identical, $\sqrt{I_{0}}$ must lie above the straight line because the droplet size must increase as the mixtures become richer in $\mathrm{H}_{2} \mathrm{O}$. The easiest way to account for the effect of the increased flow rate on $\sqrt{I_{0}}$ is to modify the straight-line relationship by multiplying each point by the ratio of the volumetric flow rates $\dot{V}_{\text {mix }} / \dot{V}_{\text {D2O }}$. In the limit of a monodisperse aerosol with constant number density, correcting by the ratio $\dot{V}_{\text {mix }} / \dot{V}_{\mathrm{D} 2 \mathrm{O}}$ is exact. The dashed line in Fig. 3 includes the volumetric flow rate correction and the agreement with the experimentally observed values improves significantly.

Finally, as long as the droplets are well mixed, i.e., there is no surface enrichment of either species, the value of $\left\langle r^{6}\right\rangle$ is not affected by the composition of the droplets-only the value of $N$ is. Thus, we can directly account for the observed changes in $\left\langle r^{6}\right\rangle=r_{g}^{6} \exp \left(18 \ln ^{2} \sigma_{r}\right)$ as a function of composition, and this is the dot-dash line in Fig. 3. This corrected curve also agrees with the measured intercepts quite reasonably, again suggesting that the droplet composition is close to the initial condensible vapor composition, and that the value of $N$ is relatively constant. Although it is possible that large changes in composition could be compensated by simultaneous large changes in $N$, we think that this is highly unlikely. Until we have better direct experimental or modeling evidence, we will assume that $x=\tilde{y}$. We note that fast isotopic exchange between $\mathrm{H}_{2} \mathrm{O}$ and $\mathrm{D}_{2} \mathrm{O}$ also helps to ensure that $x$ is close to $\tilde{y}$.

\section{B. Nucleation rates}

With our experimental values for $N$ we are now in a position to estimate the nucleation rates as a function of composition. To do so we must first evaluate the characteristic time associated with the peak nucleation rate $\Delta t_{J_{\max }}$, as well as the corresponding temperature $T_{J_{\max }}$ and supersaturation $S_{J_{\max }}$. For the pure components, the process is identical to that used by Streletzky et al. ${ }^{7}$ and Khan et al. ${ }^{13,14}$ For the binary mixtures, we treat each mixture as a single compound with physical properties that are the molar average of the pure component values. Thus, the supersaturation at any point in the nozzle is given by

$$
S=\frac{p_{\nu}}{p_{\text {mix }}^{\infty}(T)}=\frac{p_{\mathrm{D}_{2} \mathrm{O}}+p_{\mathrm{H}_{2} \mathrm{O}}}{p_{\text {mix }}^{\infty}(T)},
$$

where $p_{\nu}$ is the total partial pressure of the condensible vapor, and $p_{\mathrm{D}_{2} \mathrm{O}}$, and $p_{\mathrm{H}_{2} \mathrm{O}}$ are the partial pressure of $\mathrm{D}_{2} \mathrm{O}$ and $\mathrm{H}_{2} \mathrm{O}$, respectively. The equilibrium vapor pressure of the mixture $p_{\text {mix }}^{\infty}(T)$ is calculated as 


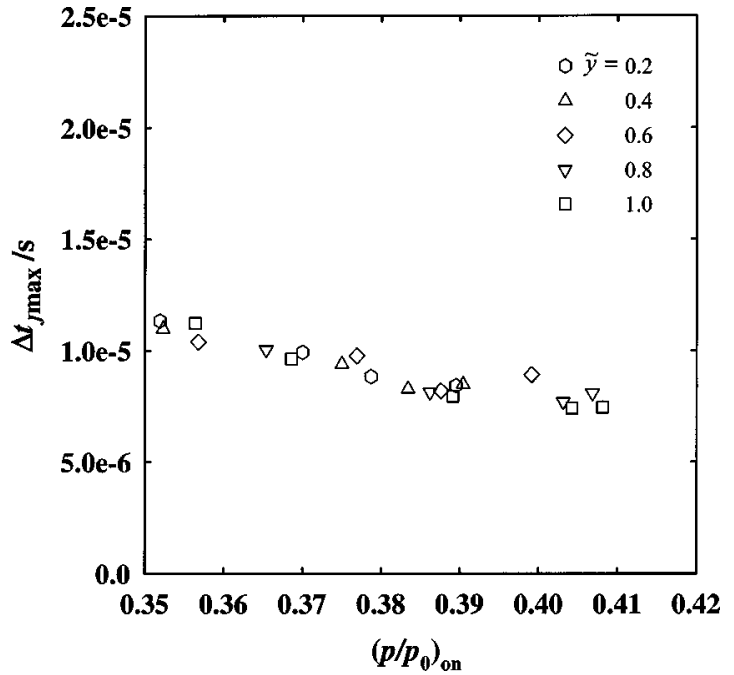

FIG. 4. The characteristic time associated with the maximum nucleation rate $\Delta t_{J_{\max }}$ varies systematically with the location of onset as characterized by $\left(p / p_{0}\right)_{\text {on }}$, but does not depend on the isotopic composition of the condensing vapor.

$$
p_{\text {mix }}^{\infty}(T)=\tilde{y} \cdot p_{\mathrm{D}_{2} \mathrm{O}}^{\infty}(T)+(1-\tilde{y}) \cdot p_{\mathrm{H}_{2} \mathrm{O}}^{\infty}(T),
$$

where $p_{\mathrm{D}_{2} \mathrm{O}}^{\infty}(T)$ and $p_{\mathrm{H}_{2} \mathrm{O}}^{\infty}(T)$ are the equilibrium vapor pressures of pure $\mathrm{D}_{2} \mathrm{O}$ and pure $\mathrm{H}_{2} \mathrm{O}$, respectively.

Our treatment here is consistent with the assumption we used to invert the pressure trace data, i.e., that the species condense together in the same ratio as in the initial condensible. Figure 4 summarizes the values of $\Delta t_{J_{\max }}$ calculated for $\mathrm{D}_{2} \mathrm{O}$ and all of the binary mixtures.

When plotted as a function of the pressure ratio at onset $\left(p / p_{0}\right)_{\text {on }}, \Delta t_{J_{\max }}$ shows no discernible dependence on composition. Because very little heat has been added to the flow at onset, we would observe similar relationships between $\Delta t_{J_{\max }}$ and the position at onset $x_{\mathrm{on}}$ or the temperature at onset $T_{\text {on }}$. For the NIST data, $\left(p / p_{0}\right)_{\text {on }}$ was always between 0.392 and 0.398 . Thus, $\Delta t_{J_{\max }}=8 \times 10^{-6} \pm 0.5 \times 10^{-6} \mathrm{~s}$, and the uncertainty in $\Delta t_{J_{\max }}$ is clearly less than $10 \%$.

At this point we note that although the pure $\mathrm{H}_{2} \mathrm{O}$ measurements gave onset values that are consistent with both previous and more recent measurements, integrating the diabatic equations to yield the other properties of the flow downstream of onset gave results that were extremely scattered and inconsistent with the other data in this series, and

TABLE III. The binary nucleation rates for $\mathrm{D}_{2} \mathrm{O}$ through pure $\mathrm{H}_{2} \mathrm{O}$ are summarized. The parentheses around the values for $\mathrm{H}_{2} \mathrm{O}$ indicate that these are extrapolated from the values observed for pure $\mathrm{D}_{2} \mathrm{O}$ and the intermediate mixtures.

\begin{tabular}{ccccccc}
\hline \hline$\tilde{y}$ & $\dot{m} / \mathrm{g} \mathrm{min}^{-1}$ & $p_{c J_{\max }} / \mathrm{kPa}$ & $f_{\exp }$ & $T_{J_{\max }} / \mathrm{K}$ & $S_{J_{\max }}$ & $J_{\max } / \mathrm{cm}^{-3} \mathrm{~s}^{-1}$ \\
\hline 1.0 & 6.47 & 0.395 & 1.37 & 229.1 & 46 & $4.1 \cdot 10^{16}$ \\
0.8 & 6.67 & 0.442 & 1.37 & 229.1 & 47 & $3.9 \cdot 10^{16}$ \\
0.6 & 6.86 & 0.454 & 1.33 & 228.6 & 47 & $3.5 \cdot 10^{16}$ \\
0.4 & 7.25 & 0.441 & 1.34 & 229.1 & 41 & $3.1 \cdot 10^{16}$ \\
0.2 & 7.51 & 0.487 & 1.34 & 229.2 & 42 & $4.0 \cdot 10^{16}$ \\
0.0 & 7.77 & $(0.549)$ & $(1.34)$ & $(229)$ & $(45)$ & $3.7 \cdot 10^{16}$ \\
\hline \hline
\end{tabular}

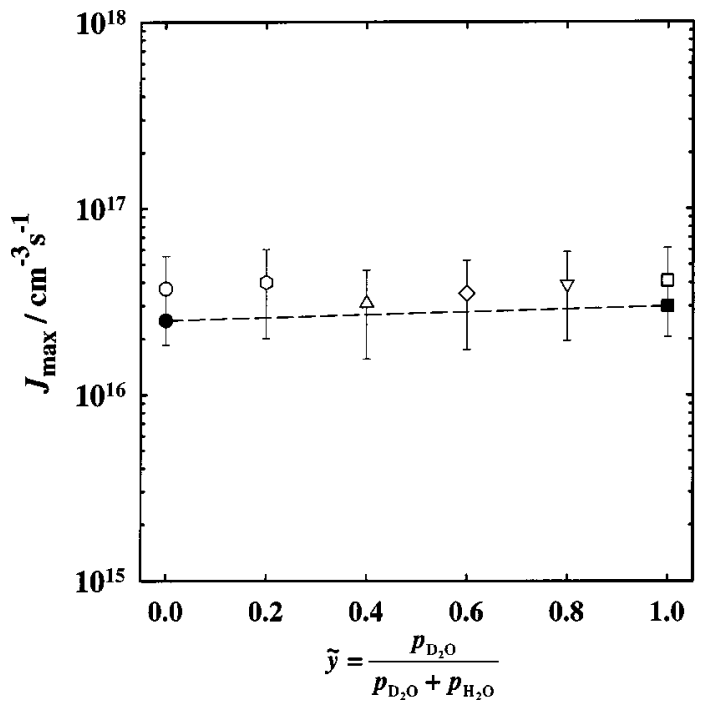

FIG. 5. The homogeneous binary nucleation rates for $\mathrm{D}_{2} \mathrm{O}-\mathrm{H}_{2} \mathrm{O}$ mixtures are independent of the vapor phase composition, and agree well with the values predicted by the empirical temperature correction function for water nucleation rates developed by Wölk and Strey (Ref. 9).

indeed any of our other measurements. For this reason, our estimates for $\Delta t_{J_{\max }}, S_{J_{\max }}$, and $T_{J_{\max }}$ for $\mathrm{H}_{2} \mathrm{O}$ are extrapolated from the $\mathrm{D}_{2} \mathrm{O}$ and mixture data and may be less certain than the values derived for the latter.

Table III and Fig. 5 summarize the nucleation rate results.

Our first observations are that $T_{J_{\max }}=229 \mathrm{~K}$ for all of the data, and that $T_{J_{\max }}$ is consistently $0.5-1 \mathrm{~K}$ lower than the corresponding values of $T_{\text {on }}$. Thus, our nucleation rate measurements are close to isothermal. The uncertainty in $T_{J_{\max }}$, is still dominated by the uncertainty in the stagnation temperature $T_{0}$, the same way that $T_{\text {on }}$ is. As discussed in detail in our previous paper, ${ }^{8}$ it is difficult to determine the stagnation temperature of the gas more accurately than $\pm 1 \mathrm{~K}$. Second, $S_{J_{\max }}$ varies between 40 and 50 for all of the conditions examined. We estimate that the error in $S_{J_{\max }}$ is \pm 5 supersaturation units, and so even the extreme values have overlapping error bars. The uncertainty in $S_{J_{\max }}$ stems partly from uncertainty in $p_{\nu}$, but is dominated by the uncertainty in $T_{J_{\max }}$ since the equilibrium vapor pressure is an exponential function of $T$. Furthermore, we expect that there is some uncertainty associated with extrapolating either vapor pressure curve to $230 \mathrm{~K}$. Table III, therefore, also reports the values of $p_{\nu}$ corresponding to the maximum rate $p_{\nu, J_{\max }}$. Finally, we see that the maximum nucleation rates, $J_{\max }$, vary by less than $20 \%$ as a function of the isotopic composition. The uncertainty in $J_{\max }$ stems both from uncertainty in $N$ and uncertainty in $\Delta t_{J_{\max }}$. As noted above, we believe the uncertainty in $\Delta t_{J_{\max }}$ is less than $10 \%$. The uncertainty in $N$ comes from uncertainty in the absolute calibration factor for the SANS experiments (about 10\%), the fitting procedure that is used (up to 20\%), and some background drift in the SANS experiments $(5 \%)$. Our overall estimate for the uncertainty in $J$ is therefore $\sim 50 \%$. The difference in the estimated nucle- 
ation rates is less than the estimated error, and, thus, for $\mathrm{D}_{2} \mathrm{O}-\mathrm{H}_{2} \mathrm{O}$ the constant onset isotherm presented in Fig. 1(b) clearly corresponds to a critical nucleation rate isotherm.

To compare our results to literature values, we turn to the extensive $\mathrm{H}_{2} \mathrm{O}-\mathrm{D}_{2} \mathrm{O}$ nucleation rate data set of Wölk and Strey. ${ }^{9}$ At $T=230 \mathrm{~K}$, they found that $\mathrm{H}_{2} \mathrm{O}$ and $\mathrm{D}_{2} \mathrm{O}$ nucleation rates in the range $10^{6}<\mathrm{J} / \mathrm{cm}^{-3} \mathrm{~s}^{-1}<10^{9}$ were indistinguishable when plotted as a function of superaturation. Our nozzle experiments are consistent with this observation and with our earlier observation ${ }^{8}$ that the supersaturation at onset for $\mathrm{H}_{2} \mathrm{O}$ and $\mathrm{D}_{2} \mathrm{O}$ merge as $T_{\text {on }}$ approaches 230-240 K. In Fig. 5, the filled symbols correspond to the nucleation rates calculated by extrapolating Wölk and Strey's empirical nucleation rate functions for pure $\mathrm{H}_{2} \mathrm{O}$ and pure $\mathrm{D}_{2} \mathrm{O}$ to nozzle conditions. The dashed line connects the two points, and the nucleation rates for the intermediate mixtures all lie close to this line. The quantitative agreement between the experimental and extrapolated values is quite amazing. We also note that, unlike rates derived from modeling pressure trace experiments only, the nucleation rates measured in the nozzle in this way are independent of any assumptions about droplet growth laws. Accordingly, in the future we should be able to decouple the two processes by careful analysis even if they remain tightly coupled in the experiment.

\section{CONCLUSIONS}

The small angle neutron scattering (SANS) experiments of $\mathrm{D}_{2} \mathrm{O}-\mathrm{H}_{2} \mathrm{O}$ aerosols shown in Fig. 2 demonstrate that even the scattering of pure $\mathrm{H}_{2} \mathrm{O}$ aerosols can be measured. The use of $\mathrm{D}_{2} \mathrm{O}$, however, greatly facilitates the measurements, and the increased scattering length density yields more detailed and quantitative information. The quantitative analysis of the formation of $\mathrm{D}_{2} \mathrm{O}-\mathrm{H}_{2} \mathrm{O}$ aerosols in a supersonic nozzle using pressure trace measurements and SANS lets us conclude that the composition of the droplets is close to that of the initial condensible vapor. The first estimates of binary nucleation rates in a supersonic nozzle yield a value of $J_{\max }=(3.6 \pm 0.5) \cdot 10^{16} \mathrm{~cm}^{-3} \mathrm{~s}^{-1}$ independent of the isotopic composition at $T_{J_{\max }}=229 \mathrm{~K}$ and supersaturation around $S_{J_{\max }}=44$. The empirical nucleation rate functions for pure water recently developed by Wölk and Strey predict $J=3.0 \cdot 10^{16} \mathrm{~cm}^{-3} \mathrm{~s}^{-1}$ for $\mathrm{D}_{2} \mathrm{O}$ and $J=2.5 \cdot 10^{16} \mathrm{~cm}^{-3} \mathrm{~s}^{-1}$ for $\mathrm{H}_{2} \mathrm{O}$. The agreement with the nozzle experiments is remarkable and well within the experimental error of a factor 2 quoted for the nucleation pulse chamber experiments.

\section{ACKNOWLEDGMENTS}

This work was supported by the National Science Foundation, under Grants No. CHE-0097896, No. CHE-0089136, and No. INT-0089897 by the Donors of the Petroleum Research Fund administered by the American Chemical Society, and by the DAAD. We acknowledge the support of the National Institute of Standards and Technology, U.S. Department of Commerce, in providing facilities used in this work. We thank G. Wilemski and J. Barker for their assistance during the SANS experiments and valuable discussions.

\section{APPENDIX: SUMMARY OF THERMOPHYSICAL PARAMETERS}

This Appendix summarizes the thermophysical parameters of $\mathrm{D}_{2} \mathrm{O}, \mathrm{H}_{2} \mathrm{O}$, and $\mathrm{N}_{2}$. Here, $\sigma_{i}$ is the surface tension, $\rho_{i}$ the density, $p_{i}^{\infty}$ the equilibrium vapor pressure, $\kappa_{i}$ the compressibility. $T$ is the temperature in $[\mathrm{K}], t$ the temperature in $\left[{ }^{\circ} \mathrm{C}\right], T_{c}$ the critical temperature, $p_{c}$ the critical pressure, $M$ is the molecular weight, $C_{p}$ is the constant pressure heat capacity of the gas, and $C_{p \ell}$ is the constant pressure heat capacity for the liquid.

$$
\begin{aligned}
& \text { 1. } \mathrm{D}_{2} \mathrm{O} \\
& \sigma_{\mathrm{D}_{2} \mathrm{O}} /(\mathrm{mN} / \mathrm{m})^{20} \\
& \rho_{\mathrm{D}_{2} \mathrm{O}} /\left(\mathrm{g} / \mathrm{cm}^{3}\right)^{9} \\
& =93.6635+0.009133 \cdot T^{\prime}-0.000275 \cdot T^{\prime 2} \\
& =0.09 \cdot \tanh (x)+0.847 \cdot t_{r}^{0.33}+0.338 \\
& x=\frac{T-231}{51.5} \quad t_{r}=\frac{T_{c}-T}{T_{c}} \\
& p_{\mathrm{D}_{2} \mathrm{O}}^{\infty}(T)(\mathrm{Pa})^{21,22} \\
& =p_{c} \cdot \exp \left\{\frac{T_{c}}{T} \cdot\left(\alpha_{1} \cdot \tau+\alpha_{2} \cdot \tau^{1.9}+\alpha_{3} \cdot \tau^{2}+\alpha_{4} \cdot \tau^{5.5}+\alpha_{5} \cdot \tau^{10}\right)\right\} \\
& \alpha_{1}=-7.81583 \quad \alpha_{4}=-3.92488 \\
& \alpha_{2}=17.6012 \quad \alpha_{5}=4.19174 \\
& \alpha_{3}=-18.1747 \quad \tau=1-T / T_{c} \\
& \kappa_{\mathrm{D}_{2} \mathrm{O}} /\left(\mathrm{Pa}^{-1}\right)^{23} \\
& =10^{-11} \cdot\left(a-b t+c t^{2}-d t^{3}+e t^{4}-f t^{5}\right) \\
& a=53.5216 \quad d=8.5541 \cdot 10^{-5} \\
& b=0.4536 \quad e=5.4089 \cdot 10^{-7} \\
& c=8.7212 \cdot 10^{-3} \quad f=1.3478 \cdot 10^{-9} \\
& T_{c}=643.89 \mathrm{~K}^{21} \\
& p_{c}=21.66 \mathrm{MPa}^{21} \\
& M=20.027 \mathrm{~g} \mathrm{~mol}^{-1} \\
& C_{p}(T=298.15 \mathrm{~K})=34.25 \mathrm{~J} \mathrm{~mol}^{-1} \mathrm{~K}^{-124} \\
& C_{p \ell}(T=293.14 \mathrm{~K})=84.23 \mathrm{~J} \mathrm{~mol}^{-1} \mathrm{~K}^{-124}
\end{aligned}
$$$$
T^{\prime}=T \cdot 1.022
$$ 


\section{2. $\mathrm{H}_{2} \mathrm{O}$}

$\sigma_{\mathrm{H}_{2} \mathrm{O}} /(\mathrm{mN} / \mathrm{m})^{25,26}$
$\rho_{\mathrm{H}_{2} \mathrm{O}} /\left(\mathrm{g} / \mathrm{cm}^{3}\right)^{9}$

$p_{\mathrm{H}_{2} \mathrm{O}}^{\infty}(T)(\mathrm{Pa})^{9}$

$\kappa_{\mathrm{H}_{2} \mathrm{O}} /\left(\mathrm{Pa}^{-1}\right)^{27}$

$T_{c}=647.15 \mathrm{~K}^{25}$

$M=18.016 \mathrm{~g} \mathrm{~mol}^{-1}$

$C_{p}(T=298.15 \mathrm{~K})=33.60 \mathrm{~J} \mathrm{~mol}^{-1} \mathrm{~K}^{-124}$

$C_{p \ell}(T=293.14 \mathrm{~K})=75.99 \mathrm{~J} \mathrm{~mol}^{-1} \mathrm{~K}^{-124}$

$$
\begin{aligned}
& =93.6635+0.009133 \cdot T-0.000275 \cdot T^{2} \\
& =0.08 \cdot \tanh (x)+0.7415 \cdot t_{r}^{0.33}+0.32 \\
& x=\frac{T-225}{46.2} \quad t_{r}=\frac{T_{c}-T}{T_{c}} \\
& =\exp (77.34491-7235.42465 / T-8.2 \cdot \ln T+0.0057113 \cdot T) \\
& =10^{-11} \cdot\left(a-b t+c t^{2}-d t^{3}+e t^{4}-f t^{5}\right) \\
& a=50.9804 \quad d=6.41785 \cdot 10^{-5} \\
& b=0.374957 \\
& e=0.343024 \cdot 10^{-6} \\
& c=7.21324 \cdot 10^{-3} \\
& f=0.684212 \cdot 10^{-9}
\end{aligned}
$$

\section{3. $\mathrm{N}_{2}$}

$M=28.013 \mathrm{~g} \mathrm{~mol}^{-1}$

$C_{\mathrm{p}\left(\mathrm{N}_{2}, T=298.15 \mathrm{~K}\right)}=29.124 \mathrm{~J} \mathrm{~mol}^{-1} \mathrm{~K}^{-124}$

${ }^{1}$ M. Kotlarchyk, S. H. Chen, J. S. Huang, and M. V. Kim, Phys. Rev. A 29, 2054 (1984).

${ }^{2}$ E. Caponetti, L. J. Magid, J. B. Hayter, and J. S. Johnson, Langmuir 2, 722 (1986).

${ }^{3}$ M. Gradzielski, D. Langevin, L. Magid, and R. Strey, J. Phys. Chem. 99, 13232 (1995).

${ }^{4}$ K. Mortensen, J. Phys.: Condens. Matter 8, A103 (1996).

${ }^{5}$ B. E. Wyslouzil, J. L. Cheung, G. Wilemski, and R. Strey, Phys. Rev. Lett. 79, 431 (1997).

${ }^{6}$ B. E. Wyslouzil, G. Wilemski, J. L. Cheung, R. Strey, and J. Barker, Phys. Rev. E 60, 4330 (1999).

${ }^{7}$ K. A. Streletzky, Y. Zvinevich, B. E. Wyslouzil, and R. Strey, J. Chem. Phys. 116, 4058 (2002).

${ }^{8}$ C. H. Heath, K. A. Streletzky, B. E. Wyslouzil, J. Wölk, and R. Strey, J. Chem. Phys. 117, 6176 (2002).

${ }^{9}$ J. Wölk and R. Strey, J. Phys. Chem. B 105, 11683 (2001).

${ }^{10}$ B. E. Wyslouzil, C. H. Heath, J. L. Cheung, and G. Wilemski, J. Chem. Phys. 113, 7317 (2000).

${ }^{11} \mathrm{~S}$. Kline, personal communication (2002) and http://www.ncnr.nist.gov/ programs/sans/manuals

${ }^{12}$ G. Wilemski, Phys. Rev. E 61, 557 (2000).

${ }^{13}$ A. Kahn, C. H. Heath, U. M. Dieregsweiler, B. E. Wyslouzil, and R. Strey (unpublished).
${ }^{14}$ A. Kahn, Master thesis, WPI, Worcester, MA (2002).

${ }^{15}$ P. E. Wagner and M. Anisimov, J. Aerosol Sci. 24s1, 103 (1993).

${ }^{16}$ K. Hämeri, M. Kulmala, E. Krissinel, and G. Kodenyov, J. Chem. Phys. 105, 7683 (1996).

${ }^{17}$ V. B. Mikheev, N. S. Laulainen, S. E. Barlow, M. Knott, and I. Ford, J. Chem. Phys. 113, 3704 (2000).

${ }^{18}$ O. Glatter, in Small-Angle X-ray Scattering, edited by O. Glatter and O. Kratky (Academic, New York, 1982).

${ }^{19}$ G. Porod, in Ref. 18.

${ }^{20}$ J. J. Jasper, J. Phys. Chem. Ref. Data 1, 949 (1972).

${ }^{21}$ P. G. Hill, R. D. C. MacMillan, and V. Lee, J. Phys. Chem. Ref. Data 11, 1 (1982).

${ }^{22}$ P. G. Hill and R. D. C. MacMillan, J. Phys. Chem. Ref. Data 9, 735 (1980).

${ }^{23}$ F. J. Millero and F. K. Lepple, J. Chem. Phys. 54, 946 (1971).

${ }^{24}$ Langes Handbook of Chemistry, 14th ed., edited by J. A. Dean (McGrawHill, New York, 1992).

${ }^{25}$ Handbook of Chemistry and Physics, 72nd ed. (CRC, Boston, 1991).

${ }^{26}$ Y. Viisanan, R. Strey, and H. Reiss, J. Chem. Phys. 99, 4680 (1993).

${ }^{27}$ G. S. Kell and E. Whalley, Philos. Trans. R. Soc. London, Ser. A 258, 565 (1965). 